\title{
Ethnologies
}

\section{Autoreprésentation et hétérostigmatisation en bandes dessinées.}

\section{La vie de Pahé de Bitam à Panam.}

\section{Mouloud Boukala}

Volume 31, numéro 2, 2010

Figures noires

Black Images

URI : https://id.erudit.org/iderudit/039371ar

DOI : https://doi.org/10.7202/039371ar

Aller au sommaire du numéro

Éditeur(s)

Association Canadienne d'Ethnologie et de Folklore

ISSN

1481-5974 (imprimé)

1708-0401 (numérique)

Découvrir la revue

Citer cet article

Boukala, M. (2010). Autoreprésentation et hétérostigmatisation en bandes dessinées. La vie de Pahé de Bitam à Panam. Ethnologies, 31(2), 219-239.

https://doi.org/10.7202/039371ar

\section{Résumé de l'article}

Cet article vise à partir de trois bandes dessinées de Pahé à interroger l'espace (culturel, politique, hospitalier) suscité par le neuvième art où un auteur se départit des illusions constitutives de sa condition en se dessinant, c'est-à-dire en se pensant. Qu'apporte ce mode de figuration et d'auto-représentation contemporaine? Qui met-on dans les cases et comment ? Ces nouvelles présences visualisées sont-elles source d'identification, de fierté, de reconnaissance ? Que recouvrent ces images ? Que condensent-elles ? Que traduisent-elles de nos sociétés, de leurs latences, de leurs espérances? Dans cette recherche, la bande dessinée n'est pas envisagée comme une illustration ou une historiette allusive à la problématique de l'auto-représentation mais considérée comme un mode de connaissance capable de rendre compte des nuances, des transformations et des ruptures qui travaillent et façonnent les pratiques sociales tant individuelles que collectives.
Ce document est protégé par la loi sur le droit d'auteur. L’utilisation des services d’Érudit (y compris la reproduction) est assujettie à sa politique d'utilisation que vous pouvez consulter en ligne.

https://apropos.erudit.org/fr/usagers/politique-dutilisation/ 


\title{
Autoreprésentation et hétérostigmatisation en BANDES DESSINÉES.
}

La vie de Pahé de Bitam à Panam.

\author{
Mouloud Boukala
}

U niversitéL umière-Lyon 2

\begin{abstract}
Les images, les idées agissent les unes sur les autres, et ces actions et réactions doivent nécessairement varier avec la nature des représentations; notamment elles doivent changer sel on que les représentations qui sont ainsi mises en rapports se ressemblent ou diffèrent ou contrastent.... Mais une fois qu'un premier fonds de représentations s'est ainsi constitué, elles deviennent, pour les raisons que nous avons dites, des réalités partiellement autonomes qui vivent d'une réalité propre (Émile Durkheim 2004 : 21-43).
\end{abstract}

Toute nation se construit dans le langage et par ses images. A ppartenir à une nation revient à partager un même ensemble de perceptions structurantes. C'est ainsi que chaque citoyen peut se penser et se rattacher à autrui. "L'essence d'une nation, écrivait Ernest Renan, est que tous les individus aient beaucoup de choses en commun » (1995: 228). Ce partage d'éléments communs participe d'une manière d'être et de rendre le monde intelligible. À l'heure actuelle, dans de nombreux pays des personnes sont ob-scènes et apparaissent inintelligibles. Privées de langage et d'images, elles sont dans un état d'errance citoyenne. Lapsus de la nation, elles appartiennent à une « nation imaginée » en leur absence comme dirait Benedict A nderson (2002 : 145). L'invitation à participer à la communion imaginée leur est déniée. Les États ne cessent de se modifier sans eux. Invisible diversité ou visible indifférence? 
Ces personnes ne sont pas passives pour autant. II y a encore une dizaine d'années, trouver une bande dessinée d'un auteur africain ou antillais croquant ses contemporains rel evait de la gageure. A ujourd'hui, ce n'est le plus cas. Pour s'en convaincre, il suffit de parcourir Les rêves de Paris (M on pierre 2004), A ya de Yopougon (A bouet et Oubrerie 2006), $M$ alamine, un africain à Paris (Edimo et $M$ bumbo 2009) ou encore $A$ frica Comics. A ntologia delle migliori storie a fumetti del Premio A frica e M editerraneo (2002), cette anthologie de trente et une bandes dessinées de créateurs africains francophones, lusophones et anglophones. Q u'apportent ces nouveaux modes de figuration et d'autoreprésentation contemporaine ? Q ui met-on dans les cases et comment ${ }^{1}$ ? C es nouvelles présences visualisées sont-elles source d'identification, de fierté, de reconnaissance? Q ue recouvrent ces images? Q ue condensent-elles? Que traduisent-elles de nos sociétés, de leurs latences, de leurs espérances? Ces imageries récemment constituées marquent-elles une rupture avec d'anciennes représentations stigmatisantes? Quelles réalités en images pour quels imaginaires? A utant d'interrogations qui constituent la matière de cette note de recherche.

Toute société a besoin d'images d'elle-même où figurent le moi, le toi, le nous et l'autre. L'enjeu primordial est d'examiner quels sont les modes de visualisation retenus qui lui offrent une occasion de se voir, de se modifier et de se transformer. En vue d'apprécier ce qui est en train de se passer collectivement, notre approche vise à étudier les liens empreints de contingence et non univoques entre les représentations collectives et les pratiques à partir d'une œuvre bédégraphique originale et stimulante, celle d'un Gabonais: Pahé. Trois albums par leur qualité documentaire et leur intérêt ethnographique ont retenu ici notre attention : La vie de Pahé. 1. Bitam (2006), La vie de Pahé. 2. Panam (2008) et Dipoula. 1. M bolo (2008).

Ce travail vise à partir de ces albums à interroger l'espace (culturel, politique, hospitalier) suscité par le neuvième art où un auteur se départit des illusions constitutives de sa condition en se dessinant, c'està-dire en se pensant. Dans cette recherche, la bande dessinée n'est pas envisagée comme une illustration ou une historiette allusive à la

1. «À l'origine, les comics cultivaient un burlesque souvent cruel prenant volontiers pour cible les minorités américaines: Irlandais, $\mathrm{N}$ oirs, A llemands, Juifs. Si les Katzenjammer Kids sont la caricature d'émigrants allemands aux États- $\mathrm{U}$ nis, $\mathrm{H}$ appy $\mathrm{H}$ ooligan concerne celle des émigrants irlandais et $\mathrm{A}$ bie the A gent est un des premiers à mettre en évidence la minorité juive, sans ironie » (Moliterni et Mellot 1996: 32). 
problématique abordée, mais considérée comme un mode de connaissance capable de rendre compte des nuances, des transformations et des ruptures qui travaillent et façonnent les pratiques sociales tant individuelles que collectives. En prise avec des formes de vie apparemment emportées dans des flux incessants de mutations, cet art séquentiel du texte et de l'image se révèle à même de décrire, d'expliquer et de mettre en crise certains processus tout en proposant une alternative à des sciences humaines souvent prisonnières de carcans méthodologiques traditionnels qui privilégient le statique sur le dynamique, l'abstrait sur le concret, la structure sur le sujet. D'aucuns s'empresseront de définir la bande dessinée comme "un art mineur réservé à des mineurs où des minorités sont représentées».

Cette approche du neuvième art ne fait que reproduire l'attitude d'une certaine anthropologie qui ajuste ses observations, ses interrogations, sa méthodologie à la taille symbolique socialement accordée à son objet d'étude. A insi, dans cette perspective les sans grade ( sans papier, sans domicile, sans image, sans travail ... ) , les pauvres, les petits (socialement), ces « objets sociaux dominés» pour reprendre une expression d'A bdelmalek Sayad (2006 1 : 28), ne peuvent donner lieu qu'à une "petite » anthropologie quand il ne s'agit pas d'une anthropologie "pauvre». Ni décorative, ni ornementale, ni instrumentale, la bande dessinée ne saurait être qualifiée de littérature mineure qu'au sens donné à cet adjectif par Gilles Deleuze et Félix Guattari : " une littérature mineure n'est pas celle d'une langue mineure, plutôt celle qu'une minorité fait dans une langue majeure » (Deleuze et Guattari 1975 : 29). Elle fait subir à une langue dominante un traitement qui la fait «tendre vers ses extrêmes ou ses limites» (1975: 32). Dans les lignes subséquentes, les œuvres graphiques seront envisagées comme une manière d'être au monde et de se rendre le monde intelligible où les images ne sont pas celles de notre amnésie mais celles de l'intelligibilité de notre époque. Chaque planche, chaque vignette sera considérée comme une proposition visuelle offrant I'occasion de j(a) uger la distance critique, cette distance entre un homme et son semblable, au-delà ou en-deçà de laquelle il se produit une crise.

\section{Pahé par Pahé : le pacte autobiographique}

Patrick Essono N kouna alias «Pahé » se fait connaître au Gabon en tant que dessinateur de presse en officiant dans des journaux virulents et caustiques tels que La G riffe, Le M oustik, le journal qui pique (sous le 
pseudonyme d'Épa), La C igale enchantée ou encore au Scribouillard. Son travail gagne en visibilité grâce à son passage à la télévision au sein de la chaîne TV Plus où il croque l'actualité pendant le journal télévisé de 20 heures et où il anime "Les N'infos de Pahé », une émission satirique diffusée toutes les fins de semaine. De sa rencontre au Cameroun avec I'éditeur suisse Pierre Paquet vont naître La vie de Pahé (deux tomes) et Dipoula, albums au sein desquels Pahé est respectivement dessinateur et scénariste, puis uniquement dessinateur, le scénario étant I'œuvre de Sti.

La vie de Pahé se présente d'emblée comme un récit de vie en images et en couleurs à la première personne, où Pahé est le rédacteur, le dessinateur et le commentateur de ses propres aventures². C ette bande dessinée peut donc être qualifiée d'autobiographique dans la mesure où il y a identité de l'auteur, du narrateur et du personnage. Elle répond à la définition de l'autobiographie telle que l'a élaborée Philippe Lejeune: «un récit rétrospectif en prose qu'une personne réelle fait de sa propre existence, lorsqu'elle met l'accent sur sa vie individuelle, en particulier sur I'histoire de sa personnalité » (1975: 14).

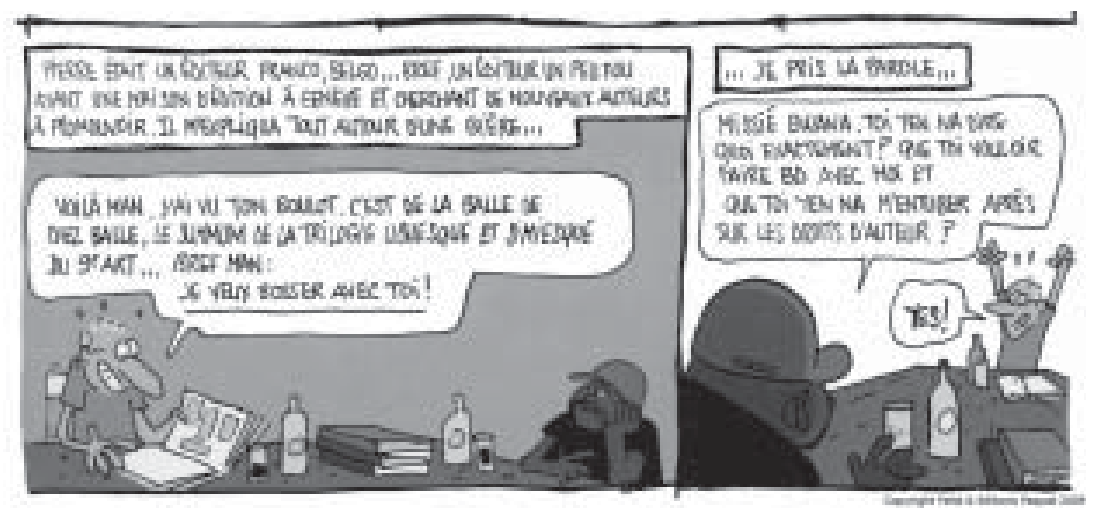

L'identité de nom entre l'auteur, le narrateur et le personnage principal s'établit de manière implicite et patente. Implicitement, à I'occasion du pacte autobiographique, puisque dès la première de couverture nous apprenons que La vie de Pahé est I'œuvre de Pahé. De manière patente par la première page de l'album où l'auteur relate la rencontre ayant présidé à la genèse de cette bande dessinée.

2. L'autobiographie se caractérise le plus souvent par l'emploi de la première personne (le narrateur étant le personnage principal). C'est ce que Gérard G enette appelle la narration « autodiégétique » dans sa classification des « voix » du récit (cf. 1972). 
L'action se déroule à "Yaoundé-21 $1^{\text {e }}$ siècle ... » et d'emblée le ton est donné. Le graphisme de Pahé, en compositions équilibrées d'une grande lisibilité, donne à apprécier la première situation mixte de l'album. A u niveau des postures, la relation est horizontale et symétrique, les deux protagonistes partageant une table et des boissons identiques. Sur le plan langagier, la réponse du principal intéressé s'effectue en parler petit-nègre. "Parler aux nègres de cette façon, c'est aller à eux, c'est les mettre à leur aise, c'est vouloir se faire comprendre d'eux, c'est les rassurer ... » rappelle Frantz Fanon (1952: 25). Pahé détourne humoristiquement ce fléchissement paternaliste dans les relations humaines où le « yes! » de l'éditeur n'est que l'affirmation d'une posture où I'on fixe, emprisonne, primitivise son interlocuteur. Dans une autre vignette, Pahé reprend de manière ironique son éditeur qui ne cesse de l'appeler «mon frère ». II existe des manières de «fraterniser » qui renferment une forme de mépris de l'autre. Elles se drapent des habits de la complaisance et de la condescendance, notamment lorsqu'un des frères vit en Suisse et l'autre au $\mathrm{G}$ abon ${ }^{3}$. Dès cette première planche, Pahé se définit comme étant simultanément une personne socialement lucide et responsable, producteur d'images (capacité à dessiner et à se dessiner) et d'un discours ( «je pris la parole», " et voilà ! Me voici sur ma table à dessin, mon cul bien calé sur une chaise prêt à vous raconter une partie de moi ... un peu de ma vie, La vie de Pahé ! »). Cette position ou affirmation par le corps et la parole où se confondent le point de vue de l'acteur et celui du spectateur le porte plus que tout autre à objectiver sa propre situation comme nous allons le voir.

Le jeu des tons, les détentes humoristiques, les pointes ironiques, les crispations orgueilleuses de Pahé sont autant de ruses sociales (ou ruses du social) face à des « rôles" » que l'auteur refuse d'endosser. Son approche n'est nullement misérabiliste et sa posture énonciative lui évite de s'instituer en porte-parole. L'originalité et la force de cette

3. Les relations qu'entretient Pahé avec Pierre Paquet s'avèrent stimulantes quant à la question de la production d'une œuvre et à son contexte de diffusion. Une entreprise autoreprésentationnelle ne fait sens qu'accompagnée d'une logique diffusionnelle. Lors d'un entretien avec Pahé nous n'avons pas manqué de I'interroger sur cet aspect : «La vie de Pahé : bande dessinée périphérique noire pour centres blancs?».

4. A u sens d'Erving Goffman 1973. 
trilogi ${ }^{5}$ reposent sur le fait que les péripéties de ce personnage au $\mathrm{G}$ abon et en France ne sont pas placées d'emblée sous l'angle des problèmes, des ennuis ou de la maladie. La vie de Pahé n'est pas estampillée « neuvième art et sida » ou « bande dessinée et conflits interethniques». Elle n'apparie pas un acteur ou un groupe social à une série de problèmes sociaux, et entre encore moins en continuité directe avec les représentations collectives existantes de l'A fricain, du N oir, de l'Émigré. L'univers de Pahé est un petit monde qui reflète - réfléchi pourrionsnous dire - celui des grands et s'adresse à eux. U ne fine observation de l'enfance s'articule à une narrativité critique. Là réside l'un des procédés les plus efficaces de l'œuvre. En quoi consiste-t-elle exactement?

\section{Le petit Pahé et les rapports de classe}

Le premier album commence, comme la plupart des autobiographies, par l'histoire de la famille et la naissance du héros, et se termine par un événement qui a changé son cadre de vie et sa destinée: une invitation par Barly Baruti au Salon du livre et de la bande dessinée de Kinshasa.

Pahé naît dans un petit village du nord du Gabon, Bitam. Son père, chef polygame du village Éboro s'efforce de faire régner un semblant d'harmonie entre ses différentes épouses, les «mouettes ». L'une d'elles le quitte emmenant à sa suite quatre filles d'un précédent mariage (Florence, Rose, Hortense et Viviane) et son dernier né, Pahé. Des années s'écoulent, Florence et Rose de retour d'Europe demandent à ce que Pahé gagne Libreville. A ccueilli par son oncle maternel Paul, notre héros découvre la vie au Carrefour Léon-M ba, un célèbre mapane, bidonville de la capitale. C'est dans ce cadre qu'il apprend comment « manger cado » (2006: 14) et qu'il pense, en compulsant un catalogue de la Redoute, que «la femme blanche ne coûte rien. $175 \mathrm{~F}$ » (2006: 16). La prochaine destination du jeune Pahé est Fala, la France où il accompagne sa sœur Rose, étudiante en médecine à Tours. S'ensuit alors toute une série d'observations et d'analyses comparatives. Le prisme du héros est gabonais. Ses descriptions sont émaillées par une forme

5. A u commencement de ce projet, La vie de Pahé a été pensée comme une trilogie dont le dernier volet «Loveman » devait dépeindre la vie amoureuse du protagoniste principal. Dans un entretien que nous a accordél'auteur, ce dernier nous a annoncé que le troisième tome, au grand regret des lecteurs, ne verrait pas le jour pour « des raisons personnelles». 
comparative où la nouveauté est estimée à l'aune d'un vécu et de références gabonais. $D$ 'emblée ce qui le frappe à son arrivée est le temps: "Un temps gris, sale. Un temps sale comme la peau de Soumou I'éléphant. A utre chose bizarre : il était sept heures du matin mais il faisait encore nuit! »(2006: 19). La course de l'avion est rapprochée de celle de la gazelle 0 pong et la manière d'aborder des contrôleurs mise en parallèle avec la technique employée par Bouky la hyène pour fondre sur sa proie. Nous sommes à Tours en 1978 et le jeune héros découvre pêle-mêle la neige, l'eau courante, le petit déjeuner, le tout à l'égout, la télévision couleur, le parc, la choucroute, les haricots, Popeye, I'absence de bruit la nuit, le supermarché $M$ ammouth et la non-valeur du franc CFA, la vie en HLM et les voisins auxquels le petit Pahé s'empresse de "faire un beau sourire comme celui du noir de la pub Banania» (2006: 31). A près l'acclimatation, le passage en classe préparatoire $(C P)$ du jeune Pahé à l'École primaire de Grandmont procède de son acculturation. Son arrivée, le fait marquant de la journée, est ainsi consignée: " En classe, il y a un nouveau. C'est un noir il vient du Gabon, c'est en A frique. II a des cheveux doux comme de la moquette... »(2006: 32).

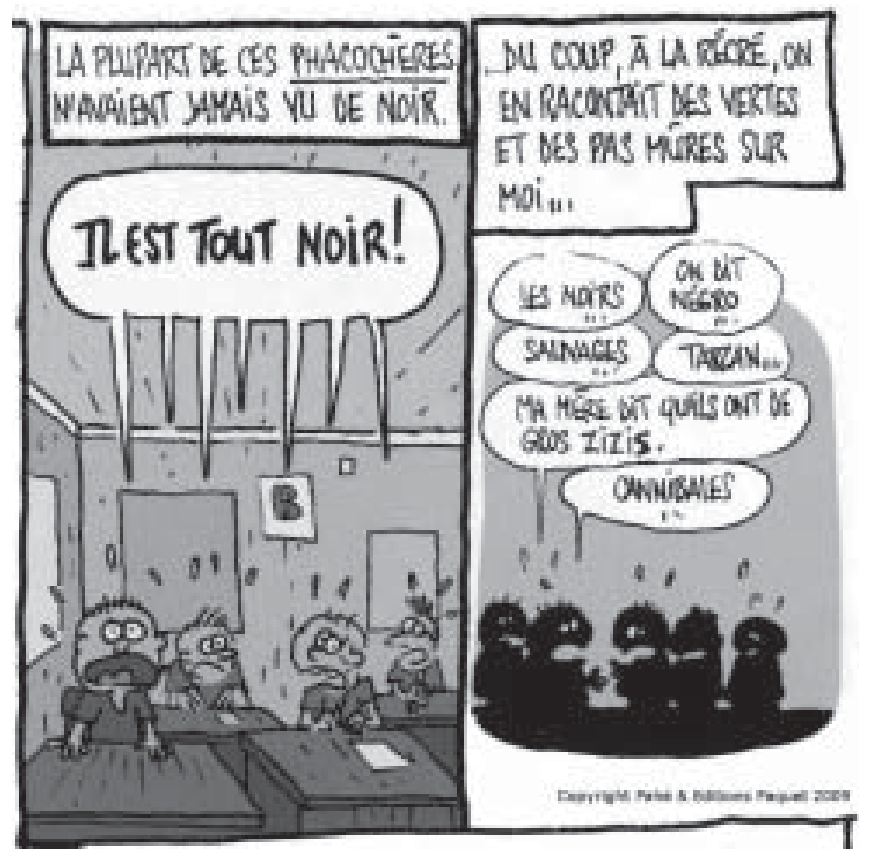


Dans ces deux albums ainsi que dans Dipoula une même trouvaille graphique est employée. Elle consiste à colorer en noir des protagonistes blancs tenant des propos ouvertement racistes ou véhiculant des préjugés ethniques. Cette manière de faire ingénieuse offre l'avantage de désamorcer la virulence de ces sacs de mots tout en dressant le portrait du « pouvoir d'intégration » de l'école. À bien des égards, sous le dessin de Pahé, I'institution éducative effraie, notamment lorsqu'il la saisit à travers les enfants, ce qu'elle en fait et ce qu'ils lui empruntent pour se construire. Sur le plan narratif, l'obtention par Rose de son diplôme de médecine signifie un retour au Gabon. Dans ce cahier d'un retour au pays natal, Pahé croque sans ménagement le PDG (Parti Démocratique G abonais) et son leader, le "grand camarade », I' « homme fort », le " guide éclairé », I' " arme du présent et du futur», ce " moustachu du sud du pays » : le président A lbert Bernard Bongo. N otre jeune héros emménage à la Sni, un quartier au sud de Libreveille peu distant de l'École publique d'O wendo dont la devise est «Discipline - Travail Réussite ». Dès lors, Pahé se livre à une fine analyse comparative des systèmes scolaires français et gabonais. Son introduction en classe par le professeur fonctionne en miroir avec la vignette précédemment reproduite. En effet, tous les écoliers s'exclament à l'unisson : "Le Blanc». Ce qualificatif ne s'appuie pas seulement sur la provenance du nouveau venu mais est également entretenu parce que Pahé « gorje», autrement dit parle avec un accent francisé. Dans cette classe surpeuplée dirigée par un enseignant alcoolique, le petit Pahé découvre, outre l'imposition du chant quotidien de I'hymne national, La Concorde, diverses violences physiques exercées à l'encontre des écoliers (coups de chicotte, coups de poings) ainsi que des punitions en tout genre (nettoyage des sanitaires, coupe des herbes hautes entourant l'école, etc.). Cette situation associée à la dégradation de ses résultats scolaires conduit notre jeune héros à changer d'établissement et à fréquenter l'école mixte conventionnée d'O wendo. Établissement initialement réservé « pour les enfants de Blancs au G abon », il est propre. Les élèves ne sont pas trois par rangs et le port de chaussures s'impose. Le cours d'histoire consacré aux ancêtres suscite nombre d'interrogations chez le petit Pahé. II ne sait qui croire entre sa nouvelle institutrice pour qui les ancêtres des $\mathrm{N}$ oirs sont égal ement les $\mathrm{G}$ aulois et son ancien professeur selon lequel il serait affilié aux Bantous et serait apparenté au grand guerrier Chaka Zoulou. La suite du premier tome est marquée par le décès de sa mère et par le départ de Pahé adulte en République Démocratique du Congo. 


\section{Tous les autres s'appellent Pahé}

C'est de la sorte, sous l'effet répété de violations dispersées, que la discrimination finit par se constituer en système, c'est-à-dire par se généraliser, par se fabriquer un système de justifications, en attendant qu'elle se découvre des vertus.... Bref, la discrimination finit par se «naturaliser » et par produire l'accoutumance qui est la marque de toutes ces « choses qui vont de soi » (Sayad $20062: 39$ ).

Si le premier opus permettait la présentation et la familiarisation du héros, le deuxième album pose avec une plus grande acuité la question de son devenir d'autant plus que le lecteur est pris entre diverses allées et venues temporelles (Pahé-adulte et Pahé-enfant) et invité à suivre notre héros au G abon, en France et en République Démocratique du Congo. Procédons chronologiquement, ce qui n'est pas le parti pris dans la bande dessinée.

A près le décès de sa mère et son entrée de sixième en poche, le jeune Pahé est envoyé à nouveau en France. II rejoint Florence, sa sœur aînée, à Villeneuve d'A scq où il entre au Collège d'enseignement supérieur (CES) du Triolo. Cette fois-ci le décalage culturel s'ancre moins sur des aspects matériels que sur l'importance que revêtent

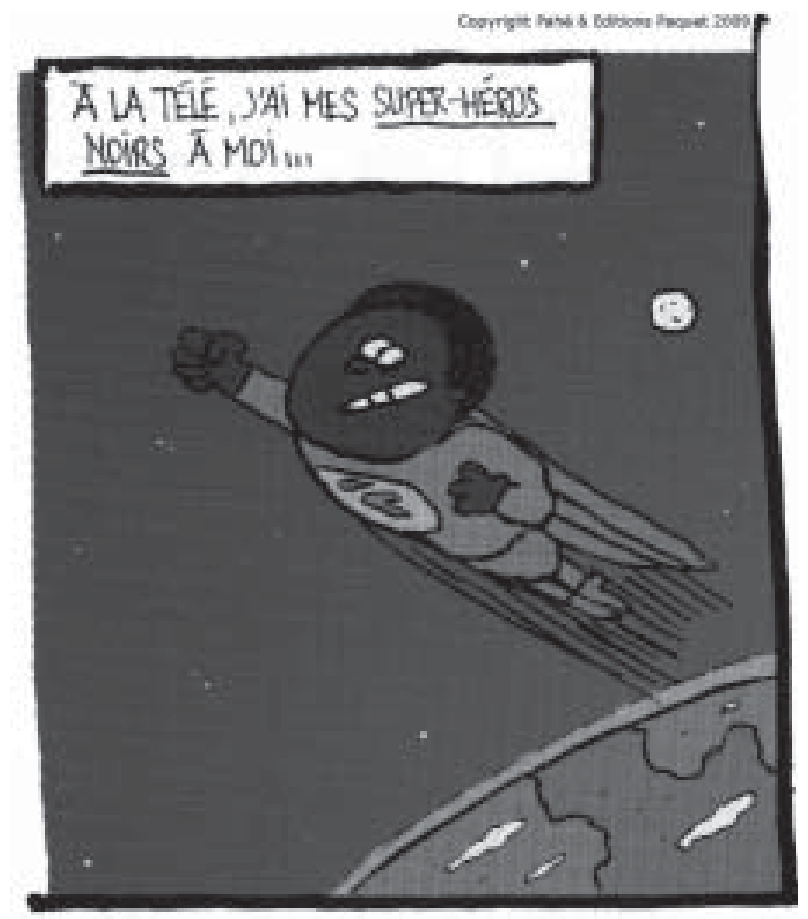


certaines représentations collectives. La peur du loup lui apparaît ridicule en comparaison d'une rencontre avec le ngongongo, un être mystique de la forêt gabonaise : "le loup ? Bof ! ... C'est un animal : on peut donc le tuer !!! » (2008: 16). C'est par le truchement de la télévision et notamment des séries américaines que le jeune Pahé effectue cette «navette incessante entre le je qui est un autre et les autres qui sont dans le je» (Morin 1956: 212).

Des projections accompagnées d'identifications façonnent son imaginaire, ce que l'auteur du C inéma ou l'homme imaginaire. Essai d'anthropologie définit par : " le ferment du travail de soi sur soi et sur la nature à travers lequel se construit et se développe la réalité de I'homme » (M orin 1956: 212). Les images télévisées suscitent des participations affectives et effectives qui modèlent son existence, et I'invitent à privilégier certains comportements et à en dédaigner d'autres. Ses super-héros sont alors Barracuda dans L'agence tous risques: " il est gentil, très fort et super costaud », H uggy les bons tuyaux dans Starsky et $\mathrm{H}$ utch et, dans une certaine mesure, M ichaël Jackson.

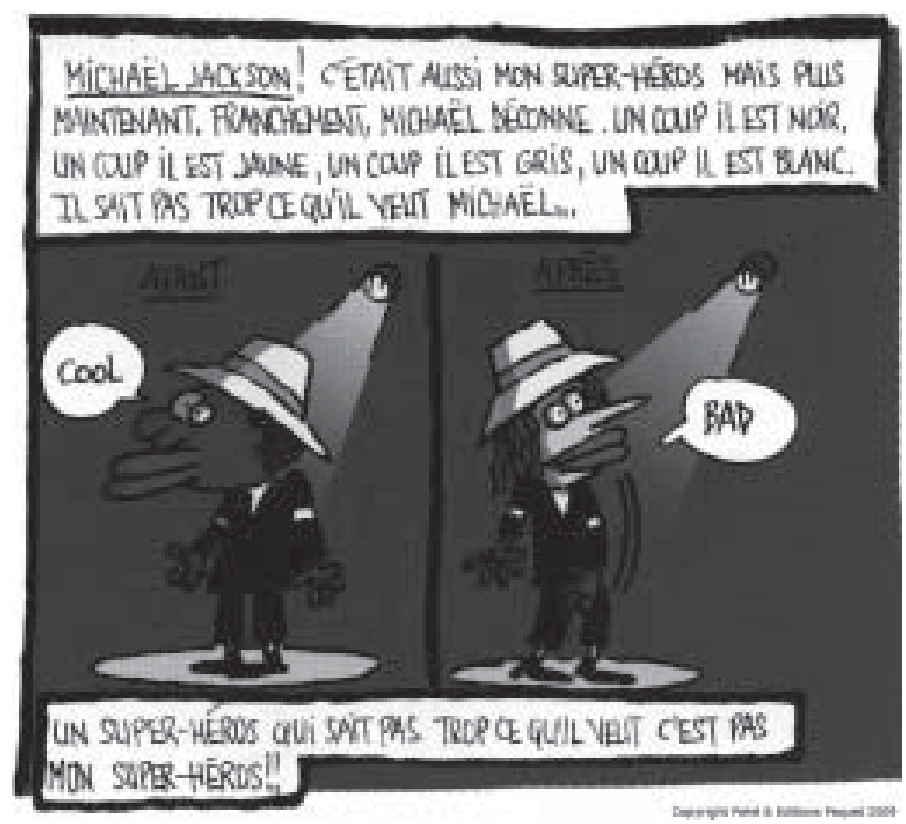


A u CES succède l'internat catholique privé N ôtre Dame de la Paix de Lille. N ous sommes en 1984 et Pahé confronte I'enseignement catholique reçu au $\mathrm{G}$ abon avec celui-ci dispensé par la sœur Bernadette. Chacune de ses prises de parole attenantes à la sphère religieuse se voit couronnée d'une " colle pour blasphème ». C'est notamment le cas Iorsqu'il affirme que« Dieu et Jésus sont des blacks!! ». N otons que les figurations noires ne sont pas uniquement mobilisées lors de séquences télévisuelles ou d'une dissertation chromato-théologique. Elles se retrouvent dans des détails les plus infimes, les plus intimes et tapissent les murs de sa chambre. Dans cette manière de s'approprier l'espace trône un poster de Sydney, une figure incontournable dans les modes de figurations et d'autoreprésentation dans les années 1980.

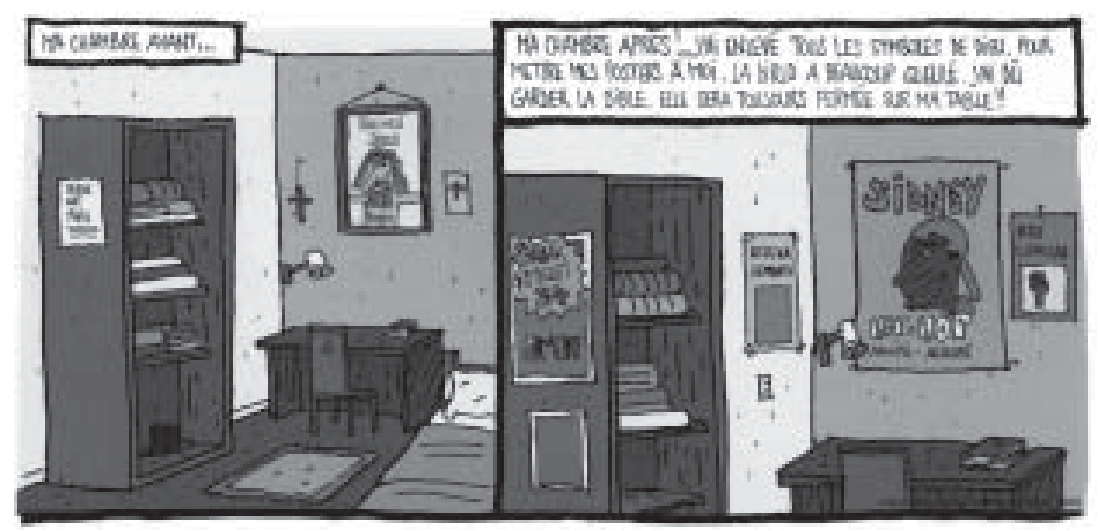

En 1984 est diffusée pour la première fois sur une chaîne française (TF1) une émission entièrement consacrée au hip-hop. D'une durée de dix minutes chaque dimanche, «H.I.P. H.O.P. » tournée au Centre Paco Rabanne est animée par Sydney qui fait alors ses débuts à la télévision. Elle rencontre un succès insoupçonné et devient rapidement un phénomène de société comme l'explique son animateur:

L'engouement est arrivé subitement en l'espace de quinze jours sur le hip-hop. La presse s'est déchaînée sur moi, sur l'émission, ça a été une folie. Je me suis vu en train de faire des doubles-pages dans VSD, des pages dans Le M onde, dans F rance Soir, dans Le N ew York Times! U n ricain m'appelle et me dit : «A ttendez, vous êtes black et vous avez une page dans le N ew York Times » J'ai dit : «A h bon! ». C 'était chose rare, M ichaël Jackson en a eu une. «Donc, vous êtes une célébrité 
noire française, qu'est-ce qui se passe ? Vous passez à la télévision française ». Je suis arrivé à N ew York, j'ai vu les « grands», M aster Flash, Meli Mel qui venaient et qui me regardaient comme si j'étais une big star alors que pour moi, au contraire, c'étaient mes stars du rap. La télé, ça a été l'explosion de tout. On ne pouvait pas prévoir que le hip-hop allait avoir une telle force. Les gens s'identifiaient enfin dans une émission. On montrait le petit M ohammed, le petit Toufik, le petit Mourad, le petit Mamadou qui devenait du jour au lendemain célèbre dans son quartier parce que, quand il rentrait, il avait fait l'émission de hip-hop ${ }^{6}$.

Précisons que l'artiste «A frica Bembata7 » (autre affiche tapissant sa chambre) fut à plusieurs reprises invitées dans H.I.P. H.O .P. U ne année plus tard, Pahé jeune adolescent est de retour au $G$ abon où il effectue son lycée puis I'Institut des Techniques A vancées en vue de devenir, selon le souhait de sa sœur, comptable. Son désintérêt manifeste pour la gestion et sa dilection pour le neuvième art participent d'un retour en France où notre héros devenu adulte poursuivra ses études à I'ISA P (Institut Supérieur d'A rt et de Publicité) de 1993 à 1996. À l'inverse d'un Tintin ou d'un A stérix, ces personnages immuables qui ne changent pas d'une page ou d'un album à l'autre, Pahé grandit. Sa réception puis sa vision de la « patrie des droits de l'homme » qu'est la France se modifie sensiblement lorsque le personnage principal atteint la majorité. A ux forces éducatives succèdent les forces de l'ordre. Dès sa descente d'avion, Paris, septembre 1993, vol A ir Gabon en provenance de Libreville, Pahé prend la mesure de l'accueil réservé par les agents chargés de la police aux frontières. Les policiers sont omniprésents et « seuls les N oirs étaient les plus visés par les contrôles ». Cette pratique à leur égard est ressentie comme autant d'épreuves humiliantes révélatrices de la suspicion en laquelle ils sont tenus. Ils ne sont pas des Français mais des suspects, des émigrés, des étrangers qui doivent montrer «patte blanche». En d'autres termes, ils ont à justifier de leur identité ( «Z'êtes sûr que c'est vous sur la photo ? Les N oirs se ressemblent tellement » lance un agent de la Police de I'A ir et des Frontières), de la régularité de leur présence, d'un domicile, d'un travail, de revenus.

Ces manières de faire sont motivées par un ensemble de normes sociales construites, acquises puis appliquées:

6. Propos extraits du documentaire de Jean-Pierre Thorn, 2003, 0 n n'est pas des marques de vélo. Paris, $M$ at films / A rte France.

7. L'orthographe exacte du créateur de la Zulu Nation est A frika Bambaataa. 
- les $\mathrm{N}$ oirs, tous semblables constituent une catégorie homogène au statut incertain ;

- ils sont surdéterminés du reste de la population française autorisant ainsi de multiples contrôles ;

- ces pratiques dépourvues d'égalité (égalité de droit et inégalité de fait) ont pour corollaire de rappeler à ces personnes qu'elles font constamment I'objet d'un travail de surveillance si ce n'est de correction consistant à réduire leurs «fautes», leurs «manquements» qu'ils accusent à l'égard de la société dans laquelle ils vivent ;

- après cet accueil, quelle que soit la régularité de sa situation, un état de légitime méfiance s'instaure et est intériorisé (quand il n'est pas extériorisé) à la vue du moindre uniforme. Des Français et des Françaises deviennent des immigrés dans leurs propre pays.

C ette relation différentielle suscite à ce non-national qu'est Pahé la remarque suivante: "A voir la nationalité française n'est pas un acquis pour tout le monde ». II nous invite à repenser les fondements d'un lien supposé établi entre le citoyen et la nation. Or, la politique actuelle ne réfléchit qu'inégalement la pluralité française. U ne même prétention à parler au nom de ce qu'est la France (une totalité inaccessible, manquante et toujours à venir) se drape en connaissances et légitime discours et pratiques politiques. Le résultat est sans équivoque : la devise nationale ( "Liberté, Égalité, Fraternité ») ne peut être sacrifiée sur l'autel de la diversité. A insi, une fiction de diversité tente d'ériger des « curiosités occasionnelles» en symboles. II est difficile de ne pas s'apercevoir du caractère fictif de cette symbolisation compensatoire. U ne étrange collusion s'exerce dans laquelle une "élite » prend sa diversité pour la réalité. $C$ ette situation leucocentrique s'avère analogue à la télévision et au cinéma. À chaque succès cinématographique national, il conviendrait de se demander: par ces images quelles sont les images que je rejette? Connaissez-vous le nom d'une seule actrice noire? $\mathrm{O}$ ui, Valérie Lemercier dans $A$ gathe Cléry, film d'Etienne Chatilliez: "Elle est blanche. Elle est raciste. Elle va devenir noire». Le noir se fait dans la salle, il n'apparaît que rarement à l'écran. II en est de même pour ceux que l'on nomme les $2 G$ ou 3G, les jeunes issus de l'immigration deuxième et troisième génération. Cependant, les représentations collectives s'altèrent peu à peu en devenant autres. Des réalisations, telles L'esquive d'A bdellatif Kechiche, Entre les murs de Laurent $\mathrm{C}$ antet, Regarde-moi d'A udrey Estrougo ou encore A ide-toi et le 
ciel t'aidera de François Dupeyron en témoignent ${ }^{8}$. L'exemple le plus prégnant demeure néanmoins l'échange de paroles entre une boulangère, campée par Karine Villard, et l'une de ses clientes dans Paris de Cédric Klapisch sorti en salles en 2008 : "Figurez-vous que j'ai découvert une chose: c'est les beurettes. J'en ai une, Kadhija, mais c'est une perle. Oui, c'est vrai, je ne vous cache pas qu'elles ont du tempérament mais dans le travail, elles sont là, elles sont droites».

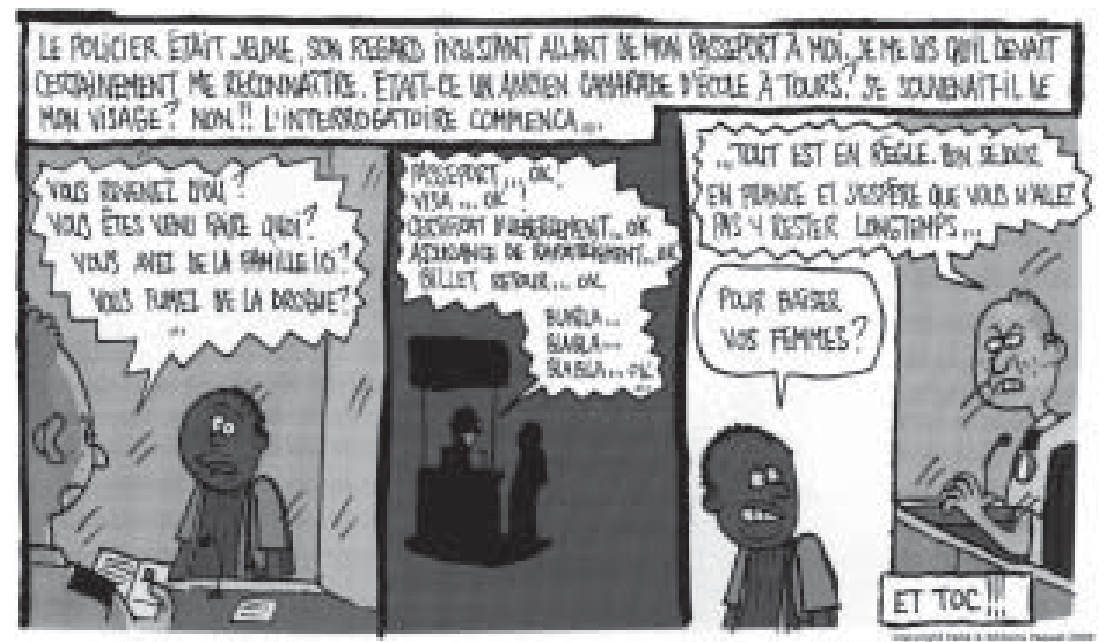

Ces situations mixtes éprouvantes impliquent la mise en œuvre de forces, d'intensités, de durées qui concourent à des opérations de sujétion et d'intimidation. Ces images témoignent de conceptions, de pratiques et d'interprétations collectives que l'état de la société favorise quand il ne les entretient et ne les perpétue pas. Le dessin de Pahé, ce Fanon de la bande dessinée ${ }^{9}$, est ici à estimer comme " une élaboration

8. «La Vie de Pahé va être adaptée mais sous le titre Le M onde de Pahé. Pour la petite histoire, on a changé pas mal de choses car en dépit du dessin qui donne l'impression d'être une BD pour enfants, le contenu est plus orienté vers les adolescents et les adultes. La série va dès lors raconter la vie d'un Pahé né en France, qui vit avec sa mère et dont le père est retourné au $G$ abon. Ils sont dans un appartement parisien. Un jour, il emmène ses amis, des petits Français, découvrir le Gabon. Le dessin animé va sortir sur France 3 en 2009, en 78 épisodes de 7 minutes, plus un épisode spécial sur le $\mathrm{G}$ abon » nous a confirmé Pahé.

9. N ous avons à l'esprit le chapitre 5 de Peau noire, masques blancs : «L'expérience vécue du Noir» (1952:88-114). 
psychologique complexe où s'intègrent, en une image signifiante, l'expérience de chacun, les valeurs et les informations circulant dans la société » (Herzlich 1969 : 23). Dans cette double vie de Pahé, I'auteur croque toute une galerie de portraits, et notamment ces "frères antillais » et ces « beaux-frères congolais ». II témoigne de la sorte d'expériences aussi contradictoires que les conditions sociales dont elles sont issues.
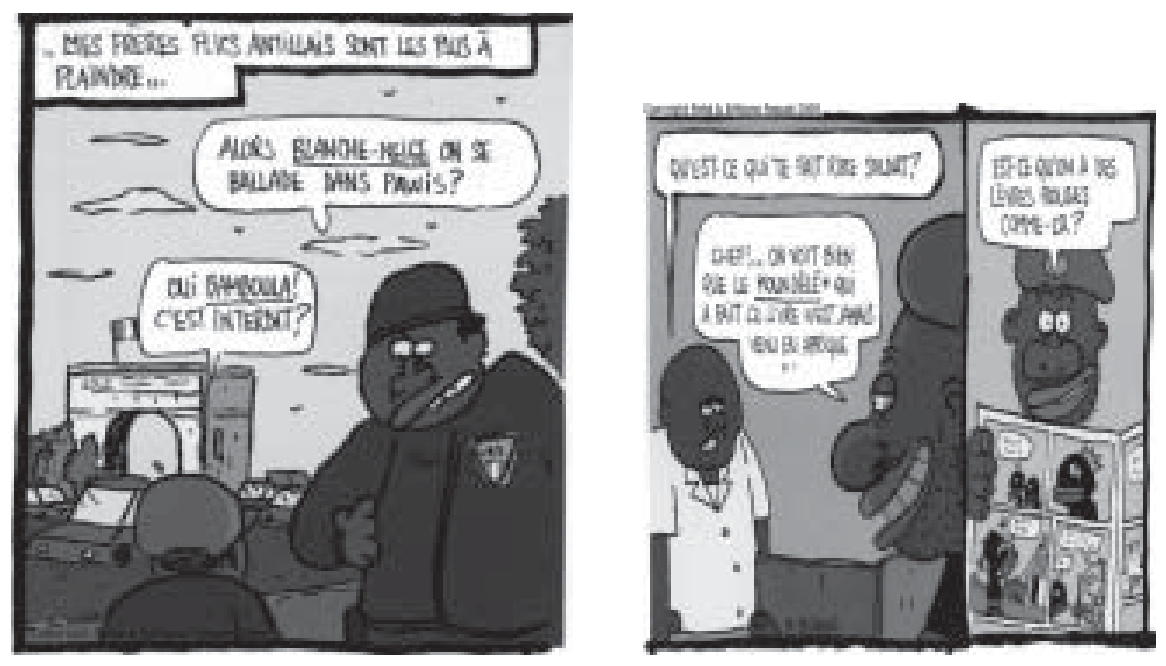

Un rappel moqueur et insidieux de sa couleur («Blanche-N eige») donne à comprendre la force du pouvoir de nomination (qui n'est autre que celui de la stigmatisation) exercé sur le groupe. A rrive un moment où les discours et représentations collectives d'une catégorie d'individus deviennent constitutifs de ces personnes comme l'avait déjà observé I'auteur de "représentations individuelles et représentations collectives ${ }^{10} »$. La représentation de la personne noire est une représentation performative, c'est-à-dire une forme visant à légitimer la distinction qu'elle a charge d'imposer. Les travaux de Pahé offrent I'occasion d'observer les contradictions engendrées en contexte africain et européen par cette performativité. Ils procèdent dans une certaine

10. Cet article fondateur de Durkheim a d'abord été publié dans La Revue de M étaphysique et de M orale (mai 1898, IV : 273-302), avant d'être repris dans Sociologie et philosophie (première édition 1924, librairie Félix A Ican). 
mesure d'une « bédéanalyse » où les bandes dessinées permettent de se penser et de se rattacher à autrui en termes profondément nouveaux. Ces œuvres constituent ce "reste », ce « résidu » qui échappe à la domination, à l'emprise de la scolarisation, au pouvoir de l'élite ${ }^{11}$ et, finalement, au visuellement correct. Elles constituent des stimulants sociaux et des alternatives là où les sociétés n'offrent plus d'issues symboliques et d'espaces à certains sujets.

\section{Dipoula : peau blanche, masques noirs}

En 2008 paraît aux éditions Paquet le premier opus de Dipoula, 1. $M$ bolo avec comme scénariste Sti, Pahé aux dessins et Callixte à la couleur. $C$ et album a nécessité un an de travail et se trouve en librairie au rayon jeunesse. II est le pendant chromatique de La vie de Pahé, là où ce dernier évoluait bien souvent dans un monde de «Blancs», les individus environnant Dipoula sont pour la plupart des « N oirs». L'unité de lecture est la page. Chacune d'entre elles porte un titre et présente les aventures et frasques d'un singulier Gabonais: Dipoula. Ce dernier est aux antipodes du « bon héros américain » au front carré surmontant un nez rectiligne, au corps athlétique et au comportement irréprochable. Petit garçon vivace flanqué d'un cochon, G ruik, il appartient à la tribu des M iénés et constitue un exclu de l'intérieur. II est albinos. Synonyme de malédiction et de mauvais œil, il est abandonné par son père dès sa naissance avant d'être recueilli dans un orphelinat administré par des sœurs catholiques «blanches». C'est essentiellement au sein de cet environnement et en compagnie de trois autres orphelins, Blazé, Cissouko et I'A méricain, que se déroulent des péripéties riches en couleurs. Dipoula n'a de cesse en tant qu'être social de vouloir appartenir au groupe. $\mathrm{Ni}$ blanc ni noir, ni vraiment du côté du M ême ni totalement du côté de l'A utre, Dipoula se situe en un lieu «bâtard » où il ne cesse de s'interroger quant à son identité. Dans sa quête de couleur, son principal adjuvant est Blazé. À deux reprises, cet acolyte tente par le truchement d'un livre de recettes mystiques dérobé à Papa Koumba de donner un teint ébène à Dipoula.

Le mythe de la potion magique n'offre aucun dédoublement. Les diverses tentatives couronnées d'échecs réitérés poussent les deux compères à s'interroger sur ce que partage Dipoula avec les Blancs et avec les $\mathrm{N}$ oirs. Le questionnement identificatoire (Blanc ou $\mathrm{N}$ oir ?) vise

11. A fin d'éviter les foudres de la censure, le travail de Pahé est consultable sur «Le blog de Pahé »: http://dipoula.paquet.li/ 


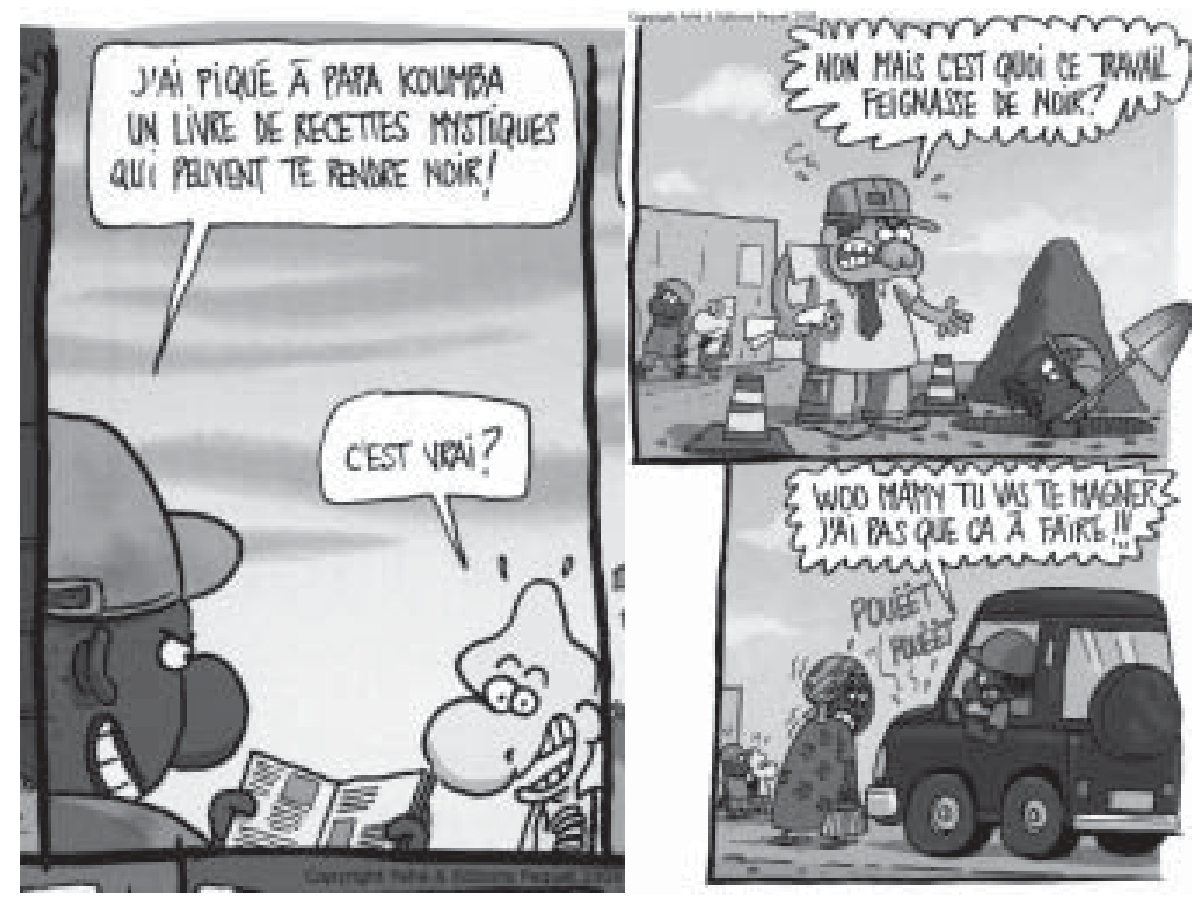

autant la sphère des valeurs que celle des pratiques. Dans leurs pérégrinations, les deux écoliers rencontrent deux adultes, un chef de chantier et un automobiliste, qui, toutes couleurs confondues ne suscitent aucun rapprochement identificatoire au protagoniste principal.

La reconnaissance et l'acceptation ou non de l'albinisme constituent I'une des composantes de cet album. Cette absence de pigmentation du héros provoque de violentes réactions de rejet. L'albinos est contagieux, son contact rend "blanc » et jaunit les cheveux. Une nouvelle venue en classe, Magalie, offre l'occasion d'apprécier le traitement de la différence. A près avoir jeté son dévolu sur cette «poupée noire», Dipoula se lance dans une entreprise empreinte de séduction. Voici le résultat.

Le traitement graphique de ces deux cases accentue la virulence de la réaction (gradation de la dominante rouge de l'arrière-fond, contour hérissé de la bulle et graisse des caractères) tout en la désamorçant. C et effet se fait avec un sens de l'économie des moyens stupéfiante. L'intensité d'une couleur chaude et l'importance de la surface colorée influencent 


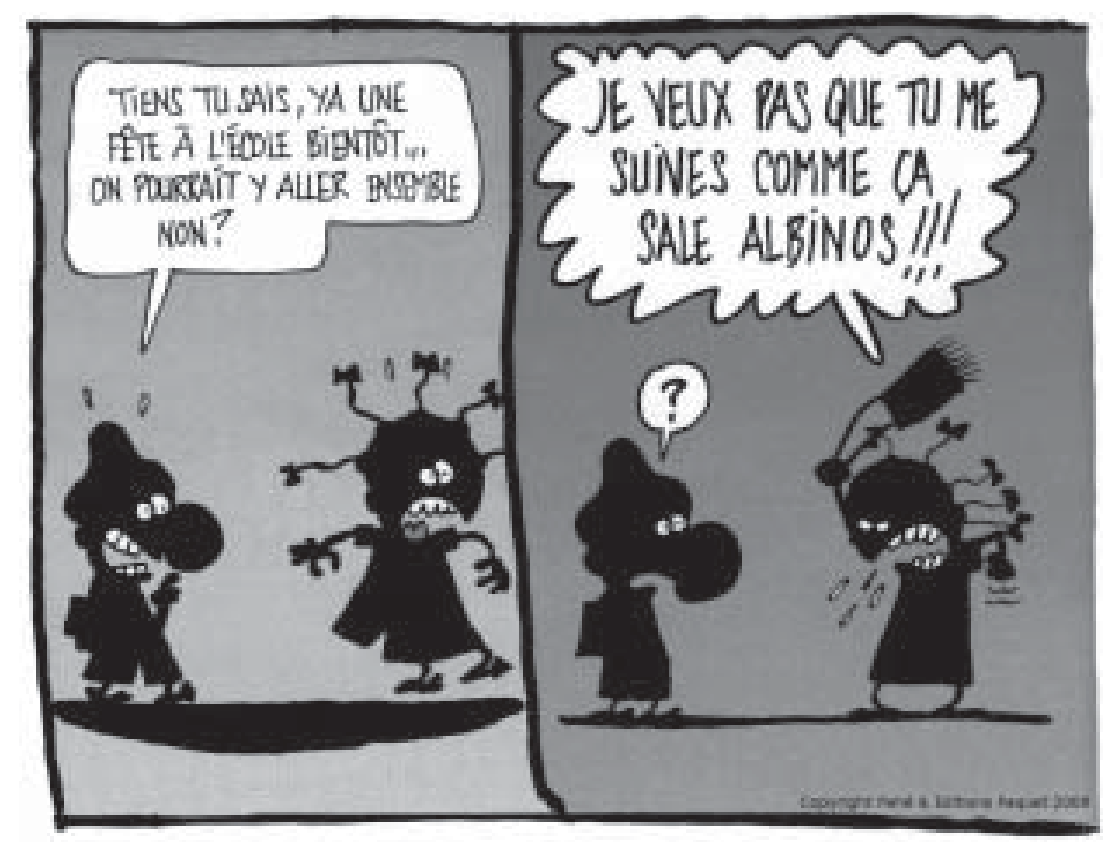

notre perception. Les personnages représentés, tous deux colorés en noir, ne partagent pas qu'un espace mais une commune appartenance. De ce contraste nait un comique d'observation. Comment une proposition de vivre-ensemble génère-t-elle un tel dégout?

Dipoula est un personnage qui autorise de nombreuses nuances et permet d'éviter certains manichéismes. Exclu au second degré, par sa condition d'orphelin et par son albinisme, les enjeux qu'il met en cause sont de nature culturelle; ils concernent essentiellement les manières de penser et d'agir, les comportements et les pratiques quotidiennes. Cette bande dessinée montre dans une certaine mesure, et sans mièvrerie, comment la différence (la couleur comme contrepoint) est suspectée de subversion et plus ou moins ouvertement accusée d'altérations culturelles. Elle donne à voir les multiples efforts déployés par un enfant pour exister dans le temps et dans l'espace au sein d'un groupe social. En ce sens, D ipoula rejoint et prolonge La vie de Pahé. La force de ces trois albums est leur «subjectivation politique » pour reprendre une expression de Jacques R ancière : cette "capacité de produire des scènes polémiques, ces scènes paradoxales qui font voir la 
contradiction de deux logiques, en posant des existences qui sont en même temps des inexistences ou des inexistences qui sont en même temps des existences» (1995: 66). La bande dessinée soulève ici le problème crucial du vivre-ensemble.

Tant que notre imaginaire ne sera pas traversé par de nouvelles représentations collectives, travaillé par des corps et des visages variés, des sociétés continueront d'ostraciser certains de leurs citoyens. Jusqu'à quand l'imaginaire d'un jeune des quartiers populaires se limitera à celui du grand frère chômeur, stagiaire à répétition, exclu de tout système et miroir d'un inéluctable « N ous étions ce que vous êtes, Vous serez qui nous sommes»? 


\section{Références}

A bouet, M arguerite et Clément O ubrerie, 2006, A ya de Yopougon. Paris, G allimard.

- - - - , 2002, A frica C omics. A ntologia delle migliori storie a fumetti del Premio A frica e M editerraneo. Bologne, Lai-momo.

A nderson, Benedict, 2002, L'imaginaire national. Réflexions sur l'origine et l'essor du nationalisme. Paris, La Découverte.

Cantet, Laurent, 2008, Entre les murs. Paris, $\mathrm{H}$ aut et court.

Chatilliez, Etienne, 2008, A gathe Cléry. Paris, Produire à Paris.

Deuleuze, Gilles et Félix Guattari,1975, Kafka. Pour une littérature mineure. Paris, M inuit.

Dupeyron, François, 2008, A ide-toi et le ciel t'aidera. Paris, A rp.

Durkheim, Emile, 2004, Sociologie et philosophie. Paris, PU F.

Edimo et M bumbo, 2009, M alamine, un africain à Paris. Vallauris, Les Enfants Rouges.

Essono Nkouna, Patrick, 2009, «Le blog de Pahé »: http:// dipoula.paquet.li/ (consulté le 21 novembre).

Estrougo, A udrey, 2006, Regarde-moi. Paris, G aumont.

Fanon, Frantz, 1952, Peau noire, masques blancs. Paris, Seuil.

Genette, Gérard, 1972, Figures III. Paris, Seuil.

Goffman, Erving, 1973, La mise en scène de la vie quotidienne. Paris, M inuit.

Herzlich, Claudine, 1969, Santé et maladie. A nalyse d'une représentation sociale. Paris, EHESS.

Kechiche, A bdellatif, 2003, L'esquive. Paris, Lola Films.

Klapisch, Cédric, 2008, Paris. Paris, Ce qui me meut Motion Picture.

Lejeune, Philippe, 1975, Le pacte autobiographique. Paris, Seuil.

Moliterni, Claude et Philippe Mellot, 1996, Chronologie de la bande dessinée. Paris, Flammarion.

M onpierre, Roland, 2004, Les rêves de Paris. Cachan, Tartamudo.

Morin, Edgar, 1956, Le cinéma ou l'homme imaginaire. Essai d'anthropologie. Paris, M inuit.

Pahé, 2006, La vie de Pahé. 1. Bitam. Genève, Paquet.

$-----, 2008, L a$ vie de Pahé. 2. Panam. Genève, Paquet.

,----- 2008 , Dipoula. 1. M bolo. G enève, Paquet.

Rancière, Jacques, 1995, La M ésentente. Politique et philosophie, Paris, Galilée.

Renan, Ernest, 1995, Q u'est-ce qu'une nation ? Et autres écrits politiques. Paris, Imprimerie nationale. 
Sayad, A bdelmalek, 2006, L'immigration ou les paradoxes de l'altérité, 1. L'illusion du provisoire. Paris, Raisons d'agir.

- - - - , 2006, L'immigration ou les paradoxes de l'altérité, 2. Les enfants illégitimes. Paris, Raisons d'agir.

Thorn, Jean-Pierre, 2003, On n'est pas des marques de vélo. Paris, M at films / A rte France. 